LANCS-TH/9919

hep-ph/9909387

(September 1999)

\title{
The abundance of moduli, modulini and gravitinos produced by the vacuum fluctuation
}

\author{
David H. Lyth \\ Department of Physics, \\ Lancaster University, \\ Lancaster LA1 4YB. U. K.
}

\begin{abstract}
Moduli, modulini and the gravitino have gravitational-strength interactions, and thermal collisions after reheating create all of them with roughly the same abundance. With their mass of order $100 \mathrm{GeV}$, corresponding to gravity-mediated supersymmetry breaking, this leads to the well-known bound $\gamma T_{\mathrm{R}} \lesssim 10^{9} \mathrm{GeV}$ on the reheat temperature, where $\gamma \leq 1$ is the entropy dilution factor. The vacuum fluctuation also creates these particles, with abundance determined by the solution of the equation for the mode function. Taking the equation in each case to be the one corresponding to a free field, we consider carefully the behaviour of the effective mass during the crucial era after inflation. It may have a rapid oscillation, which does not however affect the particle abundance. Existing estimates are confirmed; the abundance of modulini and (probably) of moduli created from the vacuum is less than from thermal collisions, but the abundance of gravitinos may be much bigger, leading to a tighter bound on $T_{\mathrm{R}}$ if supersymmetry breaking is gravitymediated.
\end{abstract}

Introduction Long-lived particle species constrain models of the early Universe, because they are cosmologically dangerous. In this note we are concerned with species that have gravitational-strength interactions. According to present ideas, these include the gravitino (spin 3/2), and species usually called moduli (spin 0) and modulini (spin 1/2).

The abundance of a given species is conveniently specified by the ratio $n / s$, where $n$ is the number density and $s$ is the entropy density, evaluated at the epoch of nucleosynthesis. A particle with a gravitational-strength decay rate, and mass $100 \mathrm{MeV}$ to $10^{5} \mathrm{GeV}$, decays before the present but after nucleosynthesis. To avoid interfering with nucleosynthesis its abundance must satisfy [1]

$$
\frac{n}{s} \lesssim 10^{-13}
$$

(The precise bound lies between $10^{-12}$ and $10^{-15}$, depending on the mass.) If supersymmetry breaking is gravity-mediated, the masses of the gravitino, moduli and modulini are all expected to lie in this range. 
A particle with gravitational-strength interactions and mass $\lesssim 100 \mathrm{MeV}$ is stable (lifetime longer than the age of the Universe). This is expected to be the case for moduli, modulini and the gravitino, if supersymmetry breaking is gauge-mediated. Any stable species which is non-relativistic at the present epoch has matter density

$$
\Omega_{X}=4.4 \times 10^{9} h^{-2} \frac{m_{X}}{1 \mathrm{GeV}} \frac{n}{s} \lesssim 0.3
$$

where $h \simeq 0.65$ is the Hubble constant in units of $100 \mathrm{~km} \mathrm{~s}^{-1} \mathrm{Mpc}^{-1}$.

Gravitinos are created by particle collisions after reheating, with abundance at nucleosynthesis [1]

$$
\left(\frac{n}{s}\right)_{\text {thermal }} \sim 10^{-13} \frac{\gamma T_{\mathrm{R}}}{10^{9} \mathrm{GeV}} .
$$

In this expression, $T_{\mathrm{R}}$ is the reheat temperature, and $\gamma^{-1} \geq 1$ is the increase in entropy, per comoving volume, between reheating and nucleosynthesis. Moduli and modulini are created with roughly the same abundance. If Eq. (1) holds for moduli, modulini or the gravitino, we arrive at the famous bound $\gamma T_{\mathrm{R}} \lesssim 10^{9} \mathrm{GeV}$.

Creation from the vacuum The vacuum fluctuation during inflation provides another mechanism for creating particles. Indeed, although there are no particles during inflation, each field has a vacuum fluctuation. During some era containing the end of inflation, the evolution of the mode functions may become non-adiabatic so that particles are created [2]. After creation stops, the number density is

$$
n=n_{*}\left(a_{*} / a\right)^{3}
$$

where $a$ is the scale factor of the Universe, and $a_{*}$ is its value at the end of inflation. We shall refer to the constant $n_{*}$ as the number density at the end of inflation, even though particle creation stops only some time later.

If the particles have only gravitational-strength interactions, or are very heavy [3, 4], they will not thermalize at reheating. The particles created by the vacuum fluctuation retain their identity, and [5] their abundance at nucleosynthesis is

$$
\begin{aligned}
\frac{n}{s} & \sim\left(\frac{n_{*}}{V^{\frac{3}{4}}}\right)\left(\frac{\gamma T_{\mathrm{R}}}{V^{\frac{1}{4}}}\right) \\
& \sim 10^{-14}\left(\frac{n_{*}}{H_{*}^{3}}\right)\left(\frac{H_{*}}{10^{14} \mathrm{GeV}}\right)\left(\frac{\gamma T_{\mathrm{R}}}{10^{9} \mathrm{GeV}}\right) .
\end{aligned}
$$

In this expression, $H_{*}$ is the practically constant value of the Hubble parameter during inflation (to be precise, the value at the end of inflation), and $V=3 M_{\mathrm{P}}^{2} H^{2}$ is the inflationary potential. The observed cosmic microwave background anisotropy requires [6] $H_{*} \lesssim 10^{14} \mathrm{GeV}\left(V_{*}^{\frac{1}{4}} \lesssim 10^{16} \mathrm{GeV}\right)$. The corresponding number density, for a stable 
non-relativistic species mass $m_{X}$, is

$$
\begin{aligned}
\Omega_{X} & \sim 10^{10}\left(\frac{n_{*}}{V_{*}^{\frac{3}{4}}}\right)\left(\frac{m_{X}}{1 \mathrm{GeV}}\right)\left(\frac{T_{\mathrm{R}}}{V_{*}^{\frac{1}{4}}}\right) \gamma \\
& \sim 10^{10}\left(\frac{n_{*}}{H_{*}^{3}}\right)\left(\frac{H_{*}}{10^{14} \mathrm{GeV}}\right)\left(\frac{m_{X}}{10^{14} \mathrm{GeV}}\right)\left(\frac{T_{\mathrm{R}}}{10^{9} \mathrm{GeV}}\right) \gamma .
\end{aligned}
$$

If $n_{*} \lesssim 10 H_{*}^{3}$, the thermal mechanism definitely dominates [5], but if $n_{*}$ is bigger the vacuum fluctuation may dominate, leading to a tighter constraint on $T_{\mathrm{R}}$. In terms of the occupation number $\left|\beta_{k}\right|^{2}$ for states with momentum $k / a$, the number density per helicity state of a given species is

$$
n=\frac{1}{2 \pi^{2}} \frac{1}{a^{3}} \int_{0}^{k_{\max }}\left|\beta_{k}\right|^{2} k^{2} \mathrm{~d} k .
$$

Significant particle creation will occur only up to some $k_{\max }$, such that $\left|\beta_{k}\right| \sim 1$ at $k=k_{\max }$, and $\left|\beta_{k}\right| \ll 1$ at bigger $k$.

For fermions, $\left|\beta_{k}\right| \leq 1$ leading to the rather firm estimate [5]

$$
n_{*} \simeq 10^{-2}\left(k_{\max } / a_{*}\right)^{3} .
$$

For scalar particles, this expression gives in general only a lower bound on $n_{*}$, because $\left|\beta_{k}\right|$ can be much bigger than 1 , but as we shall discuss it is likely to give very roughly the right order of magnitude for moduli.

For a given particle species, $k_{\max }$ is determined by the relevant mode function equation. If we are dealing with a free field, this equation corresponds to the Klein-Gordon (spin 0) Dirac (spin 1/2) or Rarita-Schwinger (spin 3/2) equation, plus constraints to eliminate unwanted degrees of freedom except in the case of scalars. The resulting equations for spin zero and spin $1 / 2$ are well known, and they have recently been given also for helicity $3 / 2$ gravitinos [7, 8, 9, 10], and for helicity $1 / 2$ gravitinos [8, 9].

In the following we consider each case in turn, paying special attention to the behaviour of the effective mass that appears in the mode function equation. It may oscillate rapidly just after inflation, but this does not affect the estimate of $k_{\max }$. In agreement with existing estimates, we find that $k_{\max } \sim a_{*} m_{*}$, where $m_{*}$ is the typical magnitude of the effective mass soon after the end of inflation. Also in agreement with these estimates we find that for moduli and modulini, $m_{*} \sim H_{*}$, while for helicity $1 / 2$ gravitinos $m_{*} \sim M$ where $M \gtrsim H_{*}$ is the mass of the field responsible for the inflaton potential.'

\footnotetext{
${ }^{1}$ Throughout this paper, we assume that oscillation of the field responsible for the inflaton potential is dominated by its the mass term. This excludes the case of inflation with a quartic potential. In that case, the abundance of particles created by the quantum fluctuation is enhanced, being the same as it would be in the case of instant reheating [8. In the case of the gravitino, we find that the advertised estimate is reduced in the unlikely event that the gravitino mass (appearing in the Rarita-Schwinger equation) is much bigger than $H$ around the end of inflation.
} 
Scalar particles The case of scalar particles, in particular moduli, is more complicated than the case of spin $1 / 2$ particles. The basic reason is that the occupation number $\left|\beta_{k}\right|$ can be much bigger than 1. As a result the number density Eq. (9) may be dominated by long wavelength modes. Related to this is the fact that the classical scalar field can have a practically homogeneous value, which in the early Universe is different from the vacuum value. In that case, the field eventually oscillates about the vacuum value, corresponding to the existence of particles with negligible momentum.

For a given field $\phi$, a crucial quantity is the effective mass-squared $m^{2}(t)$ in the early Universe. (We take the origin to be a fixed point of the symmetries so that $V=V_{0}+\frac{1}{2} m^{2} \phi^{2}$ plus higher terms.) Assuming supergravity, it is typically of order $\pm H^{2}$, until $H$ falls below the true mass $m_{X}$. Consider first the value during inflation [11, 12, 13]. Unless it is dominated by the $D$ term, the potential $V$ is of the form $\exp \left(K / M_{\mathrm{P}}^{2}\right) X$, where $K$ is the Kähler potential, and the form of $X$ does not concern us for the moment. We are assuming canonical normalisation, which means that $K=\frac{1}{2} \phi^{2}$ to leading order. As a result the potential gives a contribution of the form

$$
m_{\mathrm{pot}}^{2}=V / M_{\mathrm{P}}^{2}+\cdots .
$$

The other contribution depends on $X$, and is of order $\pm V / M_{\mathrm{P}}^{2}$. In general there is no reason to expect a cancellation, so we expect $m_{\text {pot }}^{2}=\lambda V / M_{\mathrm{P}}^{2}$, with $\lambda \sim \pm 1$. This is the only contribution to $m^{2}$ during inflation, giving a practically constant value $m_{\text {pot }}^{2}=3 \lambda H^{2}$.

Now consider the era just after inflation. The field $\chi$ responsible for $V$ oscillates, with angular frequency $M$ equal to its mass. As a result $V$ and $m_{\mathrm{pot}}^{2}$ oscillate with the same frequency. In addition, there will be a contribution [14, 9] $m_{\text {kin }}^{2}=\lambda^{\prime} \dot{\chi}^{2} / M_{\mathrm{P}}^{2}$ with $\lambda^{\prime}$ of order \pm 1 but unrelated to $\lambda$. During this era, the energy density $\rho=3 H^{2} M_{\mathrm{P}}^{2}$ and the pressure $P$ are given by

$$
\begin{aligned}
\rho & =V+\frac{1}{2} \dot{\chi}^{2} \\
P & =-V+\frac{1}{2} \dot{\chi}^{2}
\end{aligned}
$$

The quantities $V$ and $\dot{\chi}^{2}$ oscillate out of phase, so that $H^{2}$ does not oscillate. In fact, energy conservation gives $\dot{H} / H^{2}=-\frac{3}{2}(1+P / \rho)$, and according to field theory $|P| \leq \rho$. If $\lambda$ and $\lambda^{\prime}$ were equal, $m^{2}(t)$ would have the same behaviour, but as they are not it oscillates. Redefining the couplings we have

$$
m^{2} \simeq H^{2}\left(\lambda+\lambda^{\prime} \cos (M t)\right),
$$

with $|\lambda| \sim\left|\lambda^{\prime}\right| \sim 1$.

The energy in the oscillation of $\chi$ will sooner or later be spread among several particle species, through reheating or earlier during preheating (through parametric resonance). Adding together the incoherent equivalent field oscillations, we shall now get a nonoscillating contribution $m^{2}(t) \sim \pm H^{2}$. 
Assuming that $m^{2} \sim \pm H^{2}$ the cosmology of a scalar field depends crucially on the sign. If it is negative, the classical field value will be driven away from the fixed point, and will end up oscillating about its vev, corresponding to particle production that has nothing to do with the vacuum fluctuation. It is this case that is generally expected to occur for, at least some, moduli. In the case of gravity-mediated supersymmetry breaking, the estimated abundance of moduli is cosmologically dangerous. This is the usual version of the moduli problem [15]. If, on the other hand, $\mathrm{m}^{2}$ is positive during and after inflation, the classical value is always at the fixed point of the symmetries, and particle creation from the vacuum fluctuation becomes relevant. This case, corresponding to the field held at a fixed point of the symmetries, may occur for at least some of the moduli as discussed for instance in [16]. We are trying to see if the moduli problem occurs also in this case.

Before continuing with the case $m^{2} \sim H^{2}$, let us note that during inflation, it may be that $\left|m^{2}\right| \ll H^{2}$. (This is actually mandatory for the inflaton field [17], which is not however expected to be a modulus.) If that happens, then (assuming minimal coupling to gravity) the very long wavelength quantum fluctuation acts as a classical field displacement, which again eventually oscillates giving a situation essentially the same as the one in which $m^{2} \lesssim-H^{2} 18,9$, 19. 1 .

Now we briefly recall the formalism for the vacuum fluctuation (see for instance [5]). A suitably chosen mode function obeys the equation

$$
u^{\prime \prime}+\omega^{2} u=0,
$$

where

$$
\begin{aligned}
\omega^{2} & \equiv k^{2}+(a \tilde{m})^{2} \\
\tilde{m}^{2} & \equiv m^{2}-(1-6 \xi) \frac{a^{\prime \prime}}{a^{3}},
\end{aligned}
$$

and $\xi=0$ for minimal coupling to gravity. In these expressions the prime denotes differentiation with respect to conformal time $\eta$, where $\mathrm{d} \eta=\mathrm{d} t / a$. (An overdot will denote $\mathrm{d} / \mathrm{d} t$.) Einstein gravity gives

$$
\frac{a^{\prime \prime}}{a^{3}}=\frac{1}{2} H^{2}(1-3 P / \rho) .
$$

During inflation, $a^{\prime \prime} / a^{3}=2 H^{2}$, and during matter domination $a^{\prime \prime} / a^{3}=\frac{1}{2} H^{2}$.

The mode function takes on a simple form during any era in which $\omega$ satisfies the adiabatic condition. This is the condition that $\omega$ be slowly varying on the conformal timescale $\omega^{-1}$, or to be precise the conditions

$$
\left|\omega^{\prime}\right| \ll \omega^{2}, \quad\left|\omega^{\prime \prime}\right| \ll \omega^{3} .
$$

\footnotetext{
${ }^{2}$ This conclusion does not assume that $m^{2} / H^{2}$ is small also after inflation, which can hardly occur since each particle species present gives a separate contribution to it. The calculation of 5 t reaching an apparently different conclusion did not take into account the very long wavelength fluctuation.
} 
During any era in which the adiabatic condition is satisfied, approximate solutions of Eq. (15) are $u^{\text {adia }}$ and $u^{\text {adia } *}$, where

$$
u^{\text {adia }} \equiv \omega^{-\frac{1}{2}} \exp \left(-i \int_{\eta_{0}}^{\eta} \omega \mathrm{d} \eta\right),
$$

and $\eta_{0}$ is arbitrary. (Over a time interval $\ll H^{-1}$, these solutions reduce to the flat spacetime solutions.)

The adiabatic condition is satisfied at early times during inflation, and one adopts the initial condition $u=u^{\text {adia }}$, corresponding to the vacuum. At late times the adiabatic condition is again satisfied and

$$
u=\alpha u^{\text {adia }}+\beta u^{\text {adia } *},
$$

with $|\alpha|^{2}-|\beta|^{2}=1$. The occupation number is $|\beta|^{2}$, and an explicit formula is

$$
4 \omega|\beta|^{2}=\left|u^{\prime}\right|^{2}+\omega^{2}|u|^{2}-2 \omega .
$$

There is negligible particle creation if $|\beta|^{2} \ll 1$. A brief failure of the adiabatic condition need not lead to significant particle creation. At least for practical purposes, the criterion for significant particle creation is the failure of

$$
\left|\overline{\omega^{\prime}}\right| \ll \omega^{2},
$$

where the average is over a conformal time interval $\omega^{-1}$. We shall call this the weak adiabatic condition. It says that the fractional change in $\omega$ is small, in a typical conformal time interval $\omega^{-1}$. To be convinced of its relevance, one can imagine the numerical integration of Eq. (15). Alternatively, one can note that Eq. (15) describes a two-dimensional harmonic oscillator with a time-varying frequency; the initial condition corresponds to circular motion, and the final condition will also correspond to circular motion if the weak adiabatic condition is satisfied.

We want to estimate $k_{\max }$, defined as the wavenumber above which particle creation is negligible. Equivalently, $k_{\max }$ is the value of $k$, above which the weak adiabatic condition Eq. (23) is satisfied. We assume $|\xi| \lesssim 1, m^{2} \sim H^{2}$, and $\tilde{m}^{2} \sim H^{2}$. If we took $\tilde{m}^{2} / H^{2}$ to be constant, there would be no need for the average in Eq. (23). Since $a H$ peaks at the end of inflation, and varies on the inverse timescale $H$, this would give $k_{\max } \sim a_{*} H_{*}$ as advertised. In fact, $\tilde{m}^{2}$ changes at the end of inflation on the inverse timescale $M \gtrsim H$. If we ignored the average, this would give $k_{\max } \sim a_{*} \sqrt{H_{*} M}$. Restoring it, we have $\overline{\omega^{\prime}}=\left(H_{*} / M\right) \omega^{\prime}$, leading again to $k_{\max } \sim a_{*} H_{*}$.

We still have to check that Eq. (9) is dominated by the upper limit, giving $n_{*} \sim$ $10^{-2} H_{*}^{3}$. Three independent calculations [20, 5, 9] verify that this result is roughly correct if $\tilde{m}^{2} / H^{2}$ is fairly close to 1 during inflation. 1 On the other hand, $n_{*}$ is much bigger if $\tilde{m}^{2} / H_{*}^{2}$ is significantly below 1 [9, 19], due to the long wavelength effect mentioned earlier.

\footnotetext{
${ }^{3}$ Reference [20] took $|m| \ll H_{*}$ and $\xi \sim 1$, and solved the mode equation assuming $a \propto \exp \left(H_{*} t\right)$
} 
The conclusion is that the abundance of moduli created from the vacuum is less than the abundance from thermal collisions, if $\mathrm{m}^{2} / \mathrm{H}^{2}$ is fairly close to 1 during inflation, which (in magnitude) is the 'best guess' from supergravity with the potential dominated by the $F$ term. On the other hand, the abundance of moduli created from the vacuum is enhanced if $m^{2} / H^{2}$ is significantly below 1 during inflation. Related to this is the fact that a negative value $m^{2} \lesssim-H^{2}$ (during or after inflation) generates moduli through the oscillation of the classical field, with abundance typically much bigger than the abundance from thermal collisions.

Before leaving the subject of scalar particle creation, we should mention the case of a stable particle with true mass $m_{X} \gtrsim 10^{12} \mathrm{GeV}$ [3, 4]. Such a particle is a dark matte candidate (Wimpzilla) which is never in thermal equilibrium, and whose abundance from thermal collisions may be strongly suppressed. If $m_{X}$ accidentally happens to be of order $H_{*}$, one again has $k_{\max } \sim a_{*} H_{*}$, leading again to $n_{*} \sim 10^{-2} H_{*}^{3}$ if Eq. (9) is dominated by the large $k$ limit. Using a variety of approaches to the solution of the mode equation, it was found [3] that $n_{*} \sim 10^{-5} H_{*}^{3}$ (taking $\xi=\frac{1}{6}$ ) in rough agreement with this estimate. For a suitable choice of $H_{*}$ one has (Eq. (8)) a dark matter candidate. Alternatively the Wimpzillas can be created through (suppressed) thermal collisions, parametric resonance or from a first-order phase transition [1].

We end this discussion of scalar particle production by mentioning the exceptional case $\xi=1 / 6$ with $m \rightarrow 0$. The adiabatic condition is then satisfied at all times and there is no particle production, but there is no reason to expect this case to occur. The lack of particle production reflects the fact that the field equation is invariant under conformal transformations of the metric, making the expanding universe indistinguishable from flat spacetime.

Spin $1 / 2$ particles The formalism for the creation of spin $1 / 2$ particles is described for instance in [5]. A suitably normalized two-component mode function satisfies

$$
\left(\begin{array}{l}
u_{+} \\
u_{-}
\end{array}\right)^{\prime}=i\left(\begin{array}{cc}
-a m & k \\
k & a m
\end{array}\right)\left(\begin{array}{l}
u_{+} \\
u_{-}
\end{array}\right) .
$$

The evolution is unitary so that one can choose

$$
\left|u_{+}\right|^{2}+\left|u_{-}\right|^{2}=1 \text {. }
$$

during inflation, and either $a \propto t^{\frac{2}{3}}$ (matter domination) or $a \propto t^{\frac{1}{2}}$ (radiation domination) afterwards, with $u$ and $u^{\prime}$ continuous at the transition. Reference [5] took $\tilde{m}=C_{H} H$ (with $C_{H}$ a constant of order 1) and $\xi \lesssim 1$, and solved the mode equation by the same matching procedure, finding $n_{*} \sim 10^{-3.5} H_{*}^{3}$. Reference [9] made the same assumptions about $m$ and $\xi$, but solved the mode equation numerically assuming a quadratic inflation potential. The first two calculations ignore the oscillation, after inflation, of $m$ and $P / \rho$. The third includes the latter oscillation but still ignores the former. 
Defining $u \equiv u_{+}$one finds

$$
u^{\prime \prime}+\left(\omega^{2}+i(m a)^{\prime}\right) u=0,
$$

with

$$
\omega^{2}=k^{2}+(m a)^{2} .
$$

If $a m$ is slowly varying, solutions of the two-component mode equation are

$$
\begin{aligned}
& u_{ \pm}=u_{ \pm}^{\text {adia }} \equiv N_{ \pm} \exp \left(-i \int_{\eta_{0}}^{\eta} \omega \mathrm{d} \eta\right) \\
& u_{ \pm}=v_{ \pm}^{\text {adia }} \equiv N_{\mp} \exp \left(i \int_{\eta_{0}}^{\eta} \omega \mathrm{d} \eta\right),
\end{aligned}
$$

where

$$
N_{ \pm}= \pm\left(\frac{\omega \pm a m}{2 \omega}\right)^{\frac{1}{2}} .
$$

The ratio $N_{+} / N_{-}$comes from Eq. (24), and the normalization from Eq. (25). Over a time interval $\ll H^{-1}$, these are the usual flat spacetime solutions. The adiabatic condition, ensuring that they are valid, is

$$
(m a)^{\prime} \ll \omega^{2}, \quad(m a)^{\prime \prime} \ll \omega^{3} .
$$

It may be obtained by inserting the solution into Eq. (26) [21]. Equivalently, one can note that Eq. (24) requires $\left|N_{ \pm}^{\prime} / N_{ \pm}\right| \ll \omega$, while the second differentiation to get Eq. (26) requires also $\left|N_{ \pm}^{\prime \prime} / N_{ \pm}\right| \ll \omega^{2}$; the conditions on $N_{-}$are the strongest, and lead to Eq. (30).

The adiabatic condition is satisfied at early times during inflation, and at late times after inflation. Taking $u_{ \pm}=u_{ \pm}^{\text {adia }}$ at early times, corresponding to the vacuum, one finds at late times

$$
u_{ \pm}=\alpha u_{ \pm}^{\text {adia }}+\beta v_{ \pm}^{\text {adia }},
$$

with $|\alpha|^{2}+|\beta|^{2}=1$. This corresponds to

$$
u=\alpha N_{+} \exp \left(-i \int_{\eta_{0}}^{\eta} \omega \mathrm{d} \eta\right)+\beta N_{-} \exp \left(i \int_{\eta_{0}}^{\eta} \omega \mathrm{d} \eta\right) .
$$

An explicit formula for the occupation number $|\beta|^{2}$ is

$$
|\beta|^{2}=\left(\omega+a m-2 \operatorname{Im}\left(u u^{* \prime}\right)\right) /(2 \omega) .
$$

Analogously with the scalar case, a brief failure of the adiabatic condition will not cause significant particle creation, the condition for that being

$$
\left|\overline{(m a)^{\prime}}\right| \ll \omega^{2},
$$

\footnotetext{
${ }^{4}$ In [0] $(m a)^{\prime}$ is incorrectly replaced by $m a^{\prime}$. With the ansatz $m / H=$ constant that was used there, one can check that this has no effect if inflation is followed by radiation domination, and is equivalent to the replacement $m / H \rightarrow \frac{1}{2} m / H$ if inflation is followed by radiation domination.
} 
where the average is over a conformal time interval $\omega^{-1}$.

The adiabatic condition is satisfied in the limit $m \rightarrow 0$, reflecting the invariance of the Dirac equation under conformal transformations of the metric. There is no particle creation in this limit.

In contrast with the case for scalars, spin $1 / 2$ particles with gauge interactions usually have effective mass $|m| \ll H$ in the early Universe [13]. There is essentially no creation of such particles from the vacuum.

For gauge singlets, including modulini, the situation is similar to that which we discussed already for scalars and spin $1 / 2$ particles. Supergravity is expected to generate an effective mass $m \sim \pm H$, coming from several different sources just as for scalar masses [13]. The ratio $m / H$ is constant during inflation, and oscillates afterwards with angular frequency $M \gtrsim H_{*}$, before settling down to a new constant value. Applying the weak adiabatic condition Eq. (34) (not the full condition Eq. (30), which would give too big a value for $k_{\max }$ ) this leads to the advertised estimate $k_{\max } \sim a_{*} H_{*}$, and $n_{*} \sim 10^{-2} H_{*}^{3}$, leading to the conclusion that the creation of modulini from the vacuum is less efficient than the thermal creation.

Note that, in contrast with the scalar case, the abundance of spin $1 / 2$ particles is not altered if $m / H$ happens to be very small during inflation, which may happen in some models.

The gravitino mode equations At the time of writing, the study of gravitino production is just beginning [7, 8, 9, 10]. One has to consider separately the production of helicity $1 / 2$ and helicity $3 / 2$ particles, as seen by a comoving observer. If the gravitino in the early Universe can be treated as a free field, it satisfies the Rarita-Schwinger equation, with time-dependent effective mass

$$
m=e^{\frac{1}{2} K / M_{\mathrm{P}}^{2}}|W| / M_{\mathrm{P}}^{2},
$$

where $W$ is the superpotential (a holomorphic function of the complex scalar fields $\phi_{n}$ ) and $K$ is the Kähler potential (a real function of $\phi_{n}$ and $\phi_{n}^{*}$ ). In minimal supergravity, $K=\sum\left|\phi_{n}\right|^{2}$ but one expects additional terms suppressed by powers of $M_{\mathrm{P}}$. The mode equations are found by imposing constraints to eliminate unwanted degrees of freedom. For helicity 3/2, one finds [7, 8, 9, 10] the same equation, Eq. (24), as in the spin 1/2 case.

The mode equation for helicity $1 / 2$ gravitinos is more complicated [8, 9]. A suitably

\footnotetext{
${ }^{5}$ In a different context [21], $k_{\max }$ is estimated on the assumption that there is significant particle production whenever the full adiabatic condition Eq. (30) is violated. We have checked that, in that case, the same result would have been obtained using the more appropriate weak adiabatic condition Eq. (34).

${ }^{6}$ This is the well-known expression of $N=1$ supergravity. In the early Universe, it may be reasonable to consider instead [22] $N>1$.
} 
defined two-component mode function satisfies [9]

$$
\left(\begin{array}{l}
u_{+} \\
u_{-}
\end{array}\right)^{\prime}=i\left(\begin{array}{cc}
-a m & k G \\
k G^{*} & a m
\end{array}\right)\left(\begin{array}{l}
u_{+} \\
u_{-}
\end{array}\right) .
$$

The function $G$ may be written

$$
G=e^{-i \theta} A^{*},
$$

where $A=A_{1}+i A_{2}$ is the quantity defined in [8],

$$
\begin{aligned}
A_{1} & \equiv \frac{(P / \rho) H^{2}-m^{2}}{H^{2}+m^{2}} \\
A_{2} & \equiv \frac{2}{3} \frac{\dot{m}}{H^{2}+m^{2}},
\end{aligned}
$$

and

$$
\begin{aligned}
\cos \theta & \equiv-\frac{m^{2}-H^{2}}{m^{2}+H^{2}} \\
\sin \theta & \equiv \frac{2 m H}{m^{2}+H^{2}} .
\end{aligned}
$$

The evolution defined by Eq. (36) is unitary, leading to Eq. (25). The function $u=G^{-\frac{1}{2}} u_{+}$, satisfies

$$
u^{\prime \prime}+\left(\omega^{2}+i(\tilde{m} a)^{\prime}\right) u=0
$$

with

$$
\omega^{2} \equiv|A|^{2} k^{2}+(\tilde{m} a)^{2},
$$

and

$$
\begin{aligned}
\tilde{m} & =-\frac{1}{2} m-\frac{3}{2} m A_{1}-\frac{3}{2} H A_{2}-\frac{1}{2 i} \frac{\dot{A}}{A} \\
& =m+\frac{1}{2 i} \frac{\dot{G}}{G} \\
& \equiv m+\frac{1}{2}(\arg G)+\frac{1}{2} \frac{|G|}{|G|}
\end{aligned}
$$

The first expression is given in [8], while the second is given in [9]; they are equivalent by virtue of Eq. (37).ป

\footnotetext{
${ }^{7}$ To be more precise, the last terms of Eqs. (40) and (42) appear with opposite sign in [8]. The origin of this discrepancy, which may be partly due to different conventions, is not clear and will not affect order-of-magnitude estimates of the abundance. References 8, 9] give Eqs. (40)-(43) only for the special case $|A|=1$, which may be the only one of physical interest, but we have checked that they follow from Eq. (36) also in the case $|A| \neq 1$.
} 
The gravitino abundance and its cosmological consequences The formalism of the last section applies if the gravitino can be treated as a free field in the early Universe, corresponding to the Rarita-Schwinger equation. This has been demonstrated [8] only when the energy density and pressure are dominated by the oscillation of a single homogeneous scalar field, with minimal kinetic term and a potential (taken to be treelevel) dominated by the $F$ term of $N=1$ supergravity. It has been shown $[\mathbb{8}, 9]$ that in this case, $|A|=1$, so that the production of helicity $1 / 2$ gravitinos is exactly the same as production of a spin $1 / 2$ particle with mass $\tilde{m}$. At least if the magnitude of the scalar field is small on the Planck scale, this particle can be identified with the goldstino of global supersymmetry [8, 9].

In the case $|A|=1$, the last term of Eq. (42) may be written

$$
\frac{1}{2 i} \frac{\dot{A}}{A}=-\frac{1}{2} \frac{\dot{A}_{1}}{A_{2}}=\frac{3}{2} m\left(1+A_{1}\right)-\frac{\dot{w}-3 H(1+w)\left(w-A_{1}\right)}{\left[1-w^{2}+2(1+w) m^{2} / H^{2}\right]^{\frac{1}{2}}},
$$

where $w \equiv P / \rho$. During slow-roll inflation, $w$ is close to -1 with $|\dot{w}| \ll H(1+w)^{\frac{1}{2}}$, and afterwards $w$ will oscillate between \pm 1 , with angular frequency equal to the mass $M \gtrsim H_{*}$ of the field responsible for the inflaton potential. Bearing all this in mind, Eq. (45) shows that if $m \lesssim H, \tilde{m}$ increases sharply just after inflation, to a value of order $M$, in a time of order $M^{-1}$. Since we are dealing with the spin $1 / 2$ mode equation, the adiabaticity condition is $\overline{(a \tilde{m})^{\prime}} \ll \omega^{2}$, leading to the estimate $k_{\max } \sim a_{*} M$ and $n_{*} \sim 10^{-2} M^{3}$.

This is the result already anticipated [8, 9]. It is indeed reasonable to suppose that $m \lesssim H$, because according to supergravity $H^{2}=\frac{1}{3} \rho(t) / M_{\mathrm{P}}^{2}$ always receives a contribution $-m^{2}(t)$. At the present epoch this contribution is cancelled to high accuracy for some unknown reason (part of the cosmological constant problem) but during inflation there is no reason to expect that to happen, in the usual case that $H_{*}$ is bigger than the present value of the gravitino mass.

This estimate of the gravitino abundance is based on Eq. (40) for the mode function, taking $|A|=1$ so that the equation becomes the same as in the spin $1 / 2$ case. The equation $|A|=1$ (with $A$ defined by Eq. (38)) cannot be exact, because it relates the gravitino mass $m(t)$, given by Eq. (35), to the density and pressure. After reheating, or significant preheating, the density and pressure have nothing to do with the scalar fields that define $m$. One can even construct a model of inflation, albeit an unrealistic one, in which $m(t)$ can be chosen independently of the density and pressure throughout the history of the Universe; this is the model [23] which generates the inflaton potential entirely from a $D$ term.

It may be that whenever $|A|$ is different from 1, Eqs. (36) and (40) cease to be true. Let us nevertheless discuss briefly the opposite possibility, that the gravitino in the early Universe is described by the Rarita-Schwinger equation (plus constraints) with $|A| \neq 1$. This equation has well-known problems in a generic spacetime [24], but it is not clear that they exist in the special case of the Robertson-Walker Universe. The phase velocity $|A|$ associated with Eq. (40) will be less than 1, as is presumably required by causality, provided that $m$ does not vary too rapidly. Coming to the question of gravitino creation, 
the following argument suggests that the estimate $k_{\max } \sim a_{*} M$ is likely to survive. Assuming as always $m \lesssim H$, it is clear that $|\tilde{m}| a$ will have the same general behaviour as in the $|A|=1$ case, being of order $a_{*} M$ just after inflation and smaller at earlier and later epochs. Also, $|A|$ is at least roughly of order 1 just after inflation, since $P / \rho$ oscillates between \pm 1 . Choosing $k>a_{*} M$, the mode equation therefore reduces to $u^{\prime \prime}+\omega^{2} u=0$, with $\omega=|A| k$. This is the same as Eq. (15), and if Eq. (23) is satisfied the positive-frequency mode is never generated, and there is no particle creation. Eq. (23) is just

$$
k>\overline{|A|^{\prime}} /|A|^{2} .
$$

It follows that $k_{\max }$ is of order $a_{*} M$ or the maximum of $\overline{|A|^{\prime}} /|A|^{2}$, whichever is bigger. By inspection of Eq. (38), one can see that the latter quantity is typically of order $a_{*} M$ just after inflation and smaller at earlier and later times, so that indeed $k_{\max } \sim a_{*} M$.

Finally, let us look at the cosmological significance of the estimate $n_{*} \sim 10^{-2} M^{3}$. Depending on the model of inflation, $M$ can be anywhere between $H_{*}$ and (roughly) $V^{1 / 4}$, where $V^{1 / 4} \simeq\left(M_{\mathrm{P}} H_{*}\right)^{\frac{1}{2}}$ is the inflaton potential. In the extreme case $M \sim V^{\frac{1}{4}}$, Eq. (6) gives [9]

$$
\frac{n}{s} \sim 10^{-2} \frac{T_{\mathrm{R}}}{V^{\frac{1}{4}}} \gamma
$$

In the case of gravity-mediated supersymmetry breaking, $n / s \lesssim 10^{-13}$ leading to a tight bound on $T_{\mathrm{R}}$, which becomes more stringent as the scale of inflation decreases, with the nucleosynthesis bound $T_{\mathrm{R}} \gtrsim 10 \mathrm{MeV}$ imposing the constraint

$$
V^{\frac{1}{4}}>10^{9} \mathrm{GeV}
$$

Conclusion We have considered the abundance of moduli, modulini and gravitinos created from the vacuum fluctuation, paying special attention to the behaviour of the effective mass just after inflation. In agreement with previous estimates, we find that creation from the vacuum is likely to be insignificant for moduli and modulini, but may be very important for gravitinos. These estimates are based on the assumption that in the early Universe, the relevant equation is the one corresponding to a free field.

\section{Acknowledgements}

I thank Robin Tucker and Vladimir Falko for a useful conversation, and thank the organisers of COSMO99 at ICTP, Trieste, where I had useful conversations with Lev Kofman, Andrei Linde and Toni Riotto.

\section{References}

[1] S. Sarkar, Rep. on Prog. in Phys. 59 (1996) 1493. 
[2] L. Parker, Phys. Rev. 183 (1968) 562; ibid D3 (1971) 346.

[3] D. J. H. Chung, E. W. Kolb and A. Riotto, Phys. Rev. Lett. 81 (1998) 4048.

[4] D. J. H. Chung, E. W. Kolb and A. Riotto, Phys. Rev. Lett. 81 (1998) 4048; Phys. Rev. D 60 (1999) 063504.

[5] D. H. Lyth and D. Roberts, hep-ph/9609441, unpublished; D. H. Lyth, D. Roberts and M. Smith, Phys. Rev. D 57 (1998) 7120.

[6] A. R. Liddle and D. H. Lyth, Phys. Rep. 231 (1993) 1.

[7] A. L. Maroto and A. Mazumdar, hep-ph/9904206.

[8] R. Kallosh, L. Kofman, A. Linde and A. Van Proeyen, hep-th/9907124.

[9] G. F. Giudice, I. Tkachev and A. Riotto, hep-ph/9907510.

[10] M. Lemoine, hep-ph/9908333.

[11] B. A. Ovrut and P. J. Steinhardt, Phys. Lett. 133B (1983) 161; M. Dine, W. Fischler and D. Nemeschansky, Phys. Lett. 136B (1984) 169; G. D. Coughlan, R. Holman, P. Ramond and G. G. Ross, Phys. Lett. 140B (1984) 44.

[12] E. J. Copeland, A. R. Liddle, D. H. Lyth, E. D. Stewart and D. Wands, Phys. Rev. D 49 (1994) 6410; E. D. Stewart, Phys. Rev. D 51 (1995) 6847.

[13] B. de Carlos, J.A. Casas, F. Quevedo and E. Roulet, Phys. Lett. B318 (1993) 447.

[14] M. Dine, L. Randall and S. Thomas, Nucl. Phys. B458 (1996) 291.

[15] G. D . Coughlan, W. Fischler, E. W. Kolb, S. Raby and G. G. Ross, Phys. Lett. 131 (1983) B59.

[16] M. K. Gaillard, D. H. Lyth and H. Murayama, Phys. Rev. D 58 (1998) 123505.

[17] D. H. Lyth and A. Riotto, Phys. Rep. 314 (1999) 146.

[18] A. S. Goncharev, A. D. Linde and M. I. Vysotsky, Phys. Lett. 147B (1984) 27.

[19] G. Felder, L. Kofman and A. Linde, hep-ph/9909508.

[20] T. Damour and A. Vilenkin, Phys. Rev. D 53 (1996) 2981.

[21] G. F. Giudice, M. Peloso, A. Riotto and I. Tkachev, JHEP 9908:014 (1999).

[22] J. Garcia-Bellido, Phys. Lett. B418 (1998) 252.

[23] J.A. Casas and C. Munoz, Phys. Lett. B216 (1989) 37.

[24] See for instance A. Al-Saad, I. M. Benn and R. W. Tucker, Gen. Rel. Grav. 19 (1987) 1115. 